\title{
Shell pressure on the core of $\mathrm{MnO} / \mathrm{Mn}_{3} \mathrm{O}_{4}$ core/shell nanoparticles
}

\author{
N. J. O. Silva, ${ }^{*}$ M. Karmaoui, and V. S. Amaral \\ Departamento de Física and CICECO, Universidade de Aveiro, 3810-193 Aveiro, Portugal \\ I. Puente-Orench and J. Campo \\ Instituto de Ciencia de Materiales de Aragón, CSIC-Universidad de Zaragoza and Institut Laue-Langevin, BP 156, \\ F-38042 Grenoble Cedex 9, France \\ I. da Silva \\ SpLine Spanish CRG Beamline at the ESRF, 6 Rue Jules Horowitz, BP 220, 38043 Grenoble Cedex 9, France \\ and Instituto de Ciencia de Materiales de Madrid-ICMM/CSIC, Cantoblanco 28049 Madrid, Spain
}

\begin{abstract}
A. Ibarra
Laboratorio de Microscopias Avanzadas (LMA)-Instituto de Nanociencia de Aragon(INA), Universidad de Zaragoza, 50018 Zaragoza, Spain

R. Bustamante, A. Millán, and F. Palacio

Instituto de Ciencia de Materiales de Aragón, CSIC-Universidad de Zaragoza, Departamento de Física de la Materia Condensada, Facultad de Ciencias, 50009 Zaragoza, Spain
\end{abstract}

(Received 19 February 2013; published 27 June 2013)

\begin{abstract}
Here we show that spontaneous oxidation of $\mathrm{MnO}$ nanoparticles into $\mathrm{MnO} / \mathrm{Mn}_{3} \mathrm{O}_{4}$ core/shell nanoparticles has the effect of a local pressure, decreasing the $\mathrm{MnO}$ cell parameter and increasing strain, resulting in the increase of the $\mathrm{MnO}$ antiferromagnetic/paramagnetic transition temperature $T_{N}$. These effects are more severe in smaller nanoparticles.
\end{abstract}

DOI: 10.1103/PhysRevB.87.224429

PACS number(s): 75.30.Cr, 75.50.Ee, 75.50.Tt, 68.35.Gy

\section{INTRODUCTION}

A myriad of core/shell nanoparticles (NPs) have been developed and studied in the last decades aiming at the increase of functionality, stability, dispersibility, biocompatibility, and specific targeting, for instance. ${ }^{1}$ Shells can also interact synergetically with the core by changing lattice parameters, resulting in strain-engineered materials with tuned, improved, and new properties. ${ }^{2,3}$ This includes materials with strain-induced ordered structures, ${ }^{4}$ materials with tuned thermodynamic properties, ${ }^{5,6}$ and materials with tuned and new optical, ${ }^{7-13}$ electronic, ${ }^{14}$ electro-optical, ${ }^{15}$ magnetoelectric, ${ }^{16,17}$ and magnetic ${ }^{18-20}$ properties. In the case of magnetic properties, attention focused on coupling magnetic and electric properties by strain-induced multiferroic materials, ${ }^{16,17}$ on increasing anisotropy by shell-induced strain, ${ }^{19}$ and on controlling the core/shell exchange coupling by strain. ${ }^{18}$

In the context of exchange coupling, one of the systems that has received more attention in recent years is $\mathrm{MnO} / \mathrm{Mn}_{3} \mathrm{O}_{4}$ core/shell NPs, an "inverted" system where the core is antiferromagnetic (AF) and the shell is ferrimagnetic, growing epitaxially on the core. ${ }^{21-26}$ This results in a rich magnetic behavior including an enhancement of the transition temperature of the shell due to the $\mathrm{MnO}$ core, ${ }^{23}$ a change in the nature of phase transition, ${ }^{27,28}$ and an increase of the $\mathrm{MnO}$ antiferromagnetic transition temperature $T_{N}$ in the NPs when compared to bulk MnO. ${ }^{23,26,28-30}$ Some of these phenomena are poorly understood and we anticipate that controlling surface oxidation and strain starting from nonoxidized cores can give a new insight on the rich magnetic behavior of $\mathrm{MnO}$ and $\mathrm{MnO} / \mathrm{Mn}_{3} \mathrm{O}_{4} \mathrm{NPs}$.

Literature devoted to the synthesis of MnO NPs shows that the NPs are always surface oxidized to a certain extent when exposed to air, resulting in a $\mathrm{MnO} / \mathrm{Mn}_{3} \mathrm{O}_{4}$ core/shell structure, even when it is not explicitly mentioned. This is the case of MnO NPs formed by the decomposition of Mn-oleate in trioctylamine and oleic acid. The dispersion of NPs and nanopods is light green at high temperature (the color of bulk $\mathrm{MnO}$ ) becoming brown when cooled (the color of $\mathrm{Mn}_{3} \mathrm{O}_{4}$ ), indicating surface oxidation. ${ }^{31}$ The formation of brownish oxidized MnO NPs and nanopods was also reported after the decomposition of $\mathrm{H}_{2} \mathrm{O}: \mathrm{Mn}-\mathrm{ac}$ in different ratios of trioctylamine:oleic acid. ${ }^{32}$ Another example is the decomposition of $\mathrm{Mn}$-acetate in trioctylamine and oleic acid under $N_{2}$, leading to the formation of MnO NPs with sizes from 7 to $20 \mathrm{~nm} .{ }^{33}$ Again, the authors mention the formation of a black dispersion at high temperature suggesting that the $\mathrm{MnO}$ NPs are surface oxidized. In the case of MnO NPs formed by the decomposition of $\mathrm{Mn}$-fatty acid salts in the presence of free fatty acid in octadecene, $n$-eicosane, and tetracosane at $300^{\circ} \mathrm{C},{ }^{34}$ no direct evidence of oxidation is provided but the reduction of the $\mathrm{MnO}$ lattice parameter compared to that of the bulk (4.439 $\AA$ compared to $4.445 \AA$ of bulk $\mathrm{MnO}^{35}$ ) is an indirect evidence of oxidation.

Since spontaneous oxidation of $\mathrm{MnO}$ NPs is ubiquitous after air exposure, we have designed a set of experiments where MnO NPs are studied at different stages of air exposure. This allowed us to investigate the role of surface oxidation and strain on the magnetic properties of MnO NPs. 


\section{EXPERIMENT}

The systems investigated here are composed of AF $\mathrm{MnO}$ NPs with two different average sizes, obtained by the decomposition of Mn-acetate in octadecene (smaller size, labeled $\mathrm{S}$ ) and trioctylamine (larger size, labeled L). Sample S was obtained by adding $8.1 \mathrm{~g}$ of manganese(II) acetate (98\%, Aldrich) to $110 \mathrm{~mL}$ of 1 -octadecene (95\%, Sigma-Aldrich) in a $250 \mathrm{~mL}$ round bottom flask under argon. The dispersion was magnetically stirred, heated up to $290^{\circ} \mathrm{C}$ at about $6{ }^{\circ} \mathrm{C} / \mathrm{min}$, and kept at $295^{\circ} \mathrm{C}$ for $2 \mathrm{~h}$ to guarantee total decomposition. Near $290^{\circ} \mathrm{C}$ the dispersion turns light green becoming more intense with time. After cooling to room temperature, the dispersion was separated into 3 approximately equal parts. After separation, one part was always kept in argon (sample $\mathrm{S}-\mathrm{NO})$, another part $(35 \mathrm{~mL})$ was mixed with $10 \mathrm{~mL}$ of air (sample S-PO), and the third part was fully oxidized in air (sample S-Oxi). All parts were then equally washed with dichloromethane and ethanol, centrifuged, dried, and stored in argon.

Sample L was obtained by adding $7.1 \mathrm{~g}$ of manganese(II) acetate to $80 \mathrm{~mL}$ of trioctylamine (98\% Sigma-Aldrich) under argon. The dispersion was magnetically stirred, heated up to $300^{\circ} \mathrm{C}$ at about $6^{\circ} \mathrm{C} / \mathrm{min}$, and kept at $295^{\circ} \mathrm{C}$ during $1 \mathrm{~h}$ to guarantee total decomposition. The dispersion was cooled, separated into 3 parts, and further manipulated as described for sample $\mathrm{S}$. In this case, the L-PO sample was mixed in a proportion of about $27 \mathrm{~mL}$ of dispersion to $10 \mathrm{~mL}$ of air.

Transmission electron microscopy (TEM) was performed using a Jeol-2000 FXII microscope, with point-to-point and line-to-line resolutions of $0.28 \mathrm{~nm}$ and $0.14 \mathrm{~nm}$, respectively, and equipped with a INCA $200 \mathrm{X}$-Sight (Oxford Instruments) energy dispersive x-ray spectrometer (EDS). High-resolution TEM (HRTEM) was performed in a Tecnai G2-F30 field emission gun microscope with a supertwin lens and $0.2 \mathrm{~nm}$ point-to-point resolution and 0.1 line resolution. Samples for TEM observations were prepared by dispersing the nanoparticles in hexane and evaporating suspension drops on carbon-coated copper grids.

X-ray absorption spectroscopy (XAS) measurements were performed at the Spanish CRG beamline (SpLine-BM25A) at the European Synchrotron Radiation Facility (Grenoble, France). XAS spectra were recorded at Mn K edge at room temperature. The energy was set using a Si(111) double-crystal monochromator and several scans for each sample were taken to check the sample stability along the time and to improve the signal-to-noise ratio. The incoming beam was monitored using an ionization chamber, which was filled with a $50 \% \mathrm{~N}_{2}$, $50 \%$ He gas mixture. NO and PO samples were dispersed in an epoxy resin (CitoFix Powder, Struers) under argon to avoid oxidation during manipulation and then mounted in a standard sample holder. Data were collected in the fluorescence yield mode with a 13-element $\mathrm{Si}(\mathrm{Li})$ energy dispersive detector (from e2v Instruments). The $\mathrm{Mn}-\mathrm{K} \alpha$ fluorescence line was recorded with the detector placed parallel to the $\mathrm{x}$-ray electric polarization field (i.e., forming $90^{\circ}$ from the incoming $x$-ray beam). Data treatment was achieved with ATHENA software. ${ }^{36}$

dc magnetic susceptibility measurements were performed in a superconducting quantum interference device (SQUID) magnetometer model MPMS-XL, from Quantum Design, Inc., under helium atmosphere. Measurements were performed as a function of temperature with an applied field of $100 \mathrm{Oe}$ at increasing temperatures from $10 \mathrm{~K}$ to $200 / 250 \mathrm{~K}$ after cooling in the absence of the field (ZFC cooling) and cooling under $100 \mathrm{Oe}$ (FC cooling). NO and PO samples were dispersed in an epoxy resin (CitoFix Powder, Struers) under argon to avoid oxidation during manipulation and then mounted in a straw.

Powder neutron diffraction (PND) experiments were performed at the Institut Laue Langevin (ILL, France) at the diffractometers D1B and D20 using a monochromatic beam of $1.28 \AA$ and $1.36 \AA$ (high-resolution configuration), respectively. Neutron diffraction patterns were recorded as a function of the temperature from 10 to $140 \mathrm{~K}$. NO and PO samples were mounted in a standard vanadium can sealed with indium under argon. Sample L-PO was studied at D1B while all other samples were studied at D20. The contribution of the instrument to the peaks broadening was determined by the instrument resolution function built from the refinement of a $\mathrm{Na}_{2} \mathrm{Ca}_{3} \mathrm{Al}_{2} \mathrm{~F}_{14}$ standard sample, while wavelengths were refined using a $\mathrm{Si}$ standard. The analysis of the diffraction patterns was performed by Rietveld refinement using the FULLPROF package. ${ }^{37}$ The microstructure effects were treated with the integral breadth method using the Voigt model for both the instrumental and intrinsic diffraction peak shape considering a Thompson-Cox-Hastings pseudo-Voigt convoluted with axial divergence asymmetry function to describe the peak shape. The following constraints were applied to the refinements. At each temperature, the $B_{o v}$ parameter was fixed to an interpolation of the values reported in Ref. 38. In S-Oxi and L-PO samples, nuclear size at intermediate temperature was kept constant and equal to the low- and high-temperature average value. In L-Oxi and L-PO samples, strain at intermediate temperature ( 117 to $122 \mathrm{~K}$ and 50 to 130 $\mathrm{K}$, respectively) was linearly interpolated between low- and high-temperature values.

\section{RESULTS AND DISCUSSION}

The synthesis of the samples investigated here was designed to minimize surface oxidation in NO samples and increase performance in PND and HRTEM experiments, namely by avoiding the use of a surfactant and by scaling-up the synthesized samples. Both procedures lead to a poorer control of size distribution and aggregation state when compared to smaller scale and surfactant-coated NPs, as seen in transmission electron microscopy (TEM) images (Fig. 1). Anyway, the NPs obtained from the synthesis in octadecene (sample S-Oxi) have sizes in the 20 to $50 \mathrm{~nm}$ range with an average of about 30 $\mathrm{nm}$, while the oxidized NPs obtained in trioctylamine (sample L-Oxi) have sizes in the 20 to $100 \mathrm{~nm}$ range with an average of about $50 \mathrm{~nm}$. HRTEM performed on oxidized samples shows the existence of core/shell NPs, where the shell is epitaxially grown on the $\mathrm{MnO}$ core, as previously found ${ }^{22}$ (Fig. 2) and detailed next. Figure 2(a) shows a NP with the calculated fast Fourier transform (FFT) of the image in the inset. The filtered image using the $\mathrm{Mn}_{3} \mathrm{O}_{4}$ diffraction spots [marked with arrows in the inset of Fig. 2(a)] is shown in Fig. 2(b), where the location of $\mathrm{Mn}_{3} \mathrm{O}_{4}$ at the NPs' surface becomes clear. Figures 2(c)-2(e) highlight both the core-shell structure of the NPs and the epitaxy between $\mathrm{MnO}$ and $\mathrm{Mn}_{3} \mathrm{O}_{4}$. 

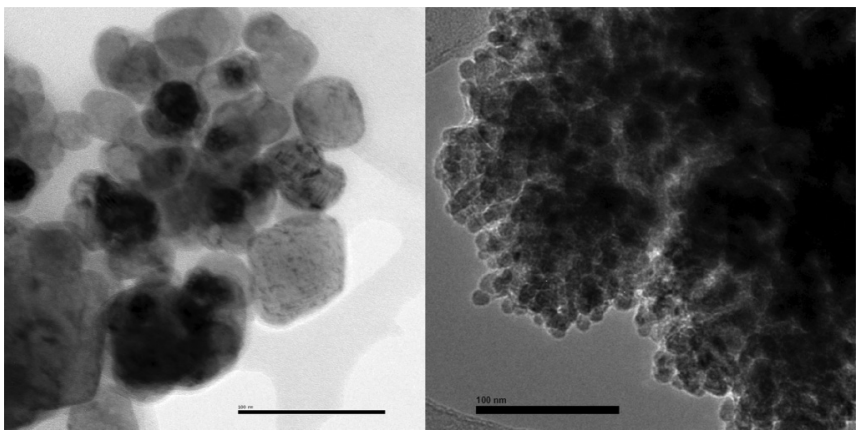

FIG. 1. TEM image of sample L-Oxi (left) and S-Oxi (right). Scale bars correspond to $100 \mathrm{~nm}$.

Figures 2(d) and 2(e) show the FFT of the NP in Fig. 2(c) calculated mainly (but not just) in the surface and core of the particle, respectively. In Fig. 2(e) (core) the spots correspond to the $\mathrm{MnO}$ cubic structure with some diffused intensity at half the reciprocal distance, while in Fig. 2(d) (surface) the spots of the $\mathrm{MnO}$ structure appear together with the $\mathrm{Mn}_{3} \mathrm{O}_{4}$ ones and perfectly oriented at about half distances in the reciprocal space.

The oxidation of the MnO NPs can be first observed by eye as the color of the suspensions changes from light green to dark green and then to brown. In an attempt at a more quantitative approach, x-ray near-edge spectroscopy (XANES) in the Mn edge was used (Fig. 3). The spectra of the L samples is similar

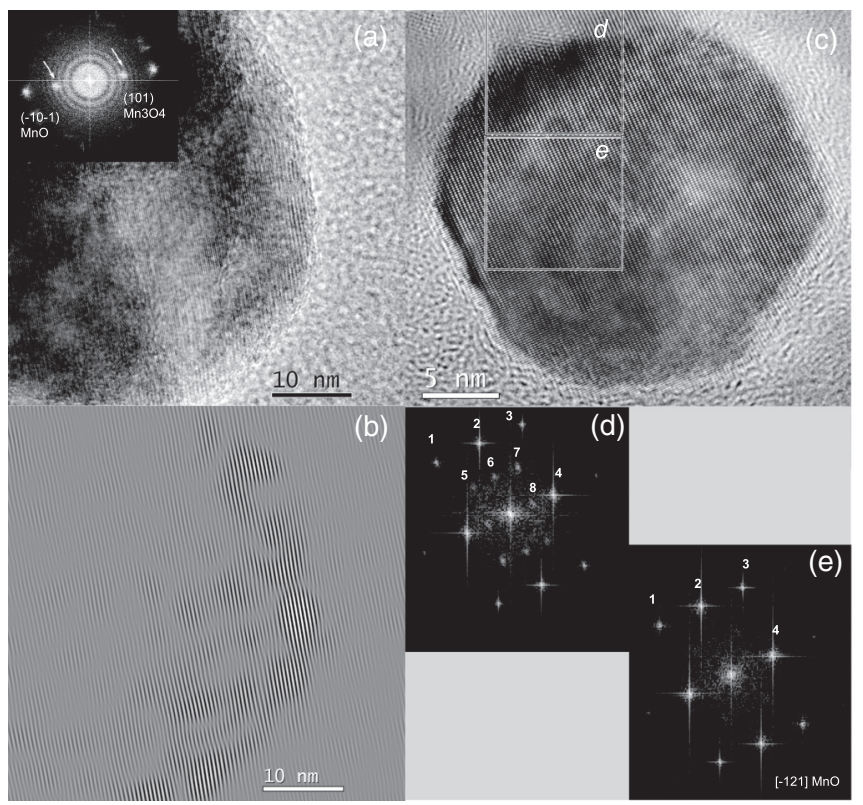

FIG. 2. (a) HRTEM image of sample L-Oxi with associated fast Fourier transform (FFT) image; (b) same image filtered at the $\mathrm{Mn}_{3} \mathrm{O}_{4}$ diffraction spots marked with arrows; (c) HRTEM image of sample L-Oxi with two highlighted regions, at surface and core, on which FT was applied; (d) surface FFT and (e) core FFT. In panel (d) and (e), spots 1, 2, 3, and 4 correspond to the (113), (202), (3-1 1), and (1-1-1) planes of the [-121] MnO zone axis, respectively. In panel (d), spots 5, 6,7 , and 8 correspond to the (113), (202), (3-11), and (1-1-1) planes of the [-121] $\mathrm{Mn}_{3} \mathrm{O}_{4}$ zone axis, respectively, considering a nondistorted cubic spinel structure with the cell parameter $a_{\text {spinel }}=2 a_{\mathrm{MnO}}$.

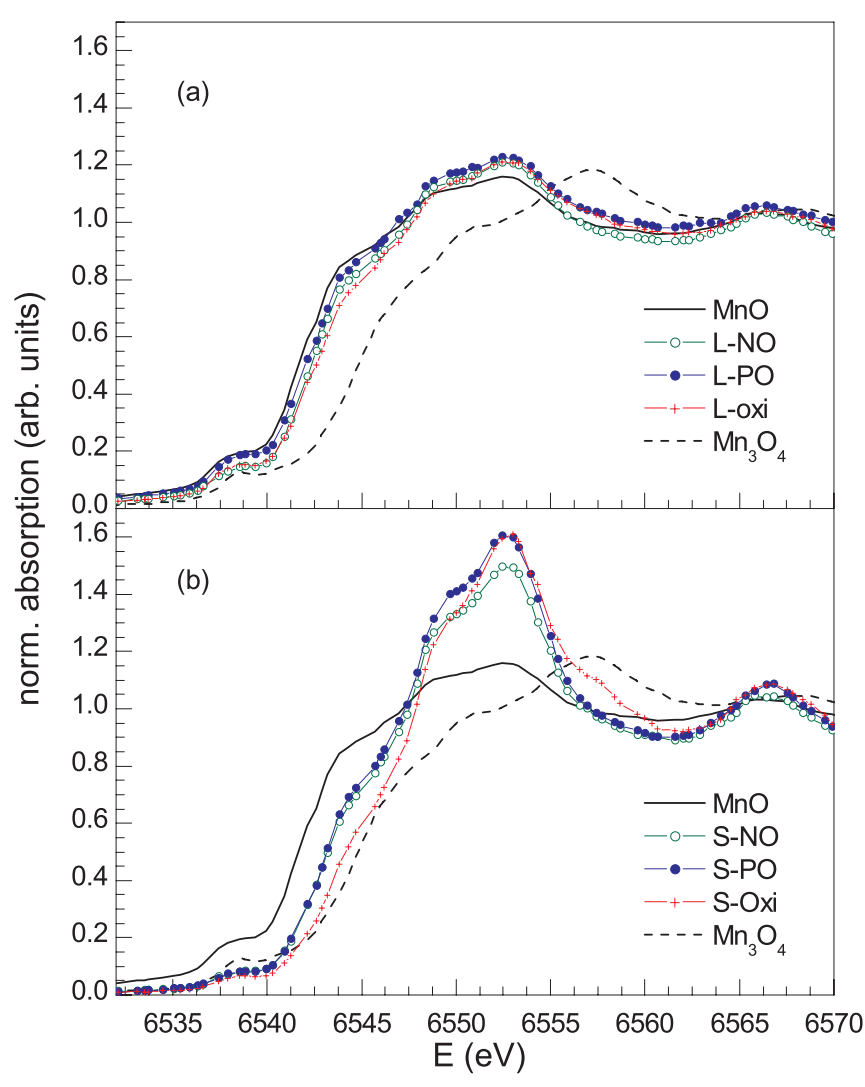

FIG. 3. (Color online) XANES spectra of $\mathrm{L}$ and $\mathrm{S}$ samples [panels (a) and (b), respectively]. The spectra of $\mathrm{MnO}$ and $\mathrm{Mn}_{3} \mathrm{O}_{4}$ samples are also shown in both panels.

to that of the $\mathrm{MnO}$ bulk sample. Changes with oxidation are quite small, although in the L-Oxi sample the edge at 6544 $\mathrm{eV}$ is shifted to higher energies as expected for more oxidized $\mathrm{Mn} .{ }^{39}$ In the case of S samples, the main features of the spectra are still those of $\mathrm{MnO}$ but different relative intensities are found, associated with different Mn environments in these samples when compared to bulk $\mathrm{MnO}$. At the same time, these differences in the relative intensities cannot be attributed to the simple existence of bulk $\mathrm{Mn}_{3} \mathrm{O}_{4}$-like $\mathrm{Mn}$ environments, showing therefore the existence of nonbulk Mn environments. Concerning the evolution of the spectra with oxidation, the S-NO and S-PO samples are similar and closer to that of $\mathrm{MnO}$, while the S-Oxi sample shows also features of $\mathrm{Mn}_{3} \mathrm{O}_{4}$, as the edge shifted to higher energy and with the appearance of a shoulder at $6557 \mathrm{eV}$. The small changes observed in the XANES spectra of $\mathrm{L}$ samples when compared to $\mathrm{S}$ samples is probably associated with the larger volume/surface ratio in the L particles that makes the XANES spectra dominated by the MnO-like contribution.

The effect of oxidation and size was further studied by dc susceptibility $(M / H, M$ being magnetization and $H$ the applied magnetic field) (Fig. 4). The temperature dependence of $M / H$ of the nonoxidized samples has the characteristic behavior of AF MnO systems, with a maximum around $120 \mathrm{~K}$ associated with an AF-paramagnetic transition and residual thermal irreversibility (measured as the difference between the FC and ZFC curves). With oxidation, this maximum becomes broader and another component with large thermal 


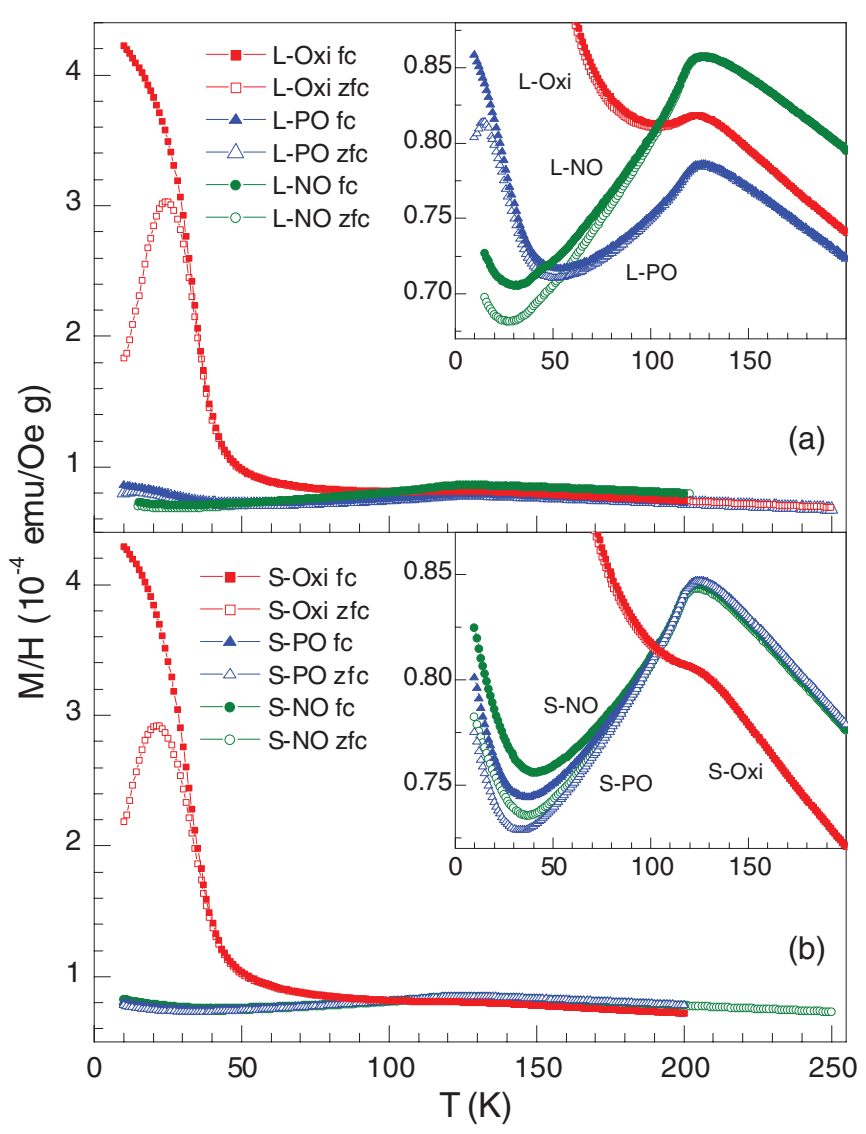

FIG. 4. (Color online) Temperature dependence of the low-field susceptibility $M / H$ of L samples [panel (a)] and S samples [panel (b)] obtained after ZFC (open symbols) and FC (full symbols) procedures. Insets show a zoom over the low $M / H$ values.

irreversibility becomes visible below $50 \mathrm{~K}$, associated with the presence of ferrimagnetic $\mathrm{Mn}_{3} \mathrm{O}_{4}$. The effect of oxidation and size on the AF-paramagnetic transition is better seen in a normalized $d(M T / H) / d T$ plot (Fig. 5), which is proportional to the magnetic specific heat in the context of mean-field theory (see, for instance, Ref. 40). In both sets of samples, it is clear that with oxidation, the peak of $d(M T / H) / d T$ and therefore the transition becomes broader, this being more severe on the high-temperature side. At the same time, with oxidation, the peak position has a small shift towards higher temperatures. Concerning size effects on the nonoxidized samples, the peak of the L-NO sample occurs at $117 \mathrm{~K}$ close to the transition temperature of bulk $\mathrm{MnO}\left(\simeq 118 \mathrm{~K}^{41}\right)$ while in the nonoxidized sample with smaller size (S-NO) the peak occurs at 115 $\mathrm{K}$. Therefore, in nonoxidized $\mathrm{MnO}$ samples, the transition temperature decreases with the decrease of size, as usually found in other magnetic NPs and attributed to finite-size effects. Also, the previous increase of $T_{N}$ reported in $\mathrm{MnO}$ NPs when compared to bulk ${ }^{23,26,28-30}$ is shown here to be associated with the spontaneous oxidation occurring in $\mathrm{MnO}$ NPs.

The possible origins of the $T_{N}$ increase in $\mathrm{MnO}$ cores (summarized in Ref. 26) were previously associated to size and surface effects with different possible causes such as (i) an enhancement of exchange interaction, ${ }^{42}$ (ii) variations in the crystal field resulting in high-spin low-spin transitions and/or

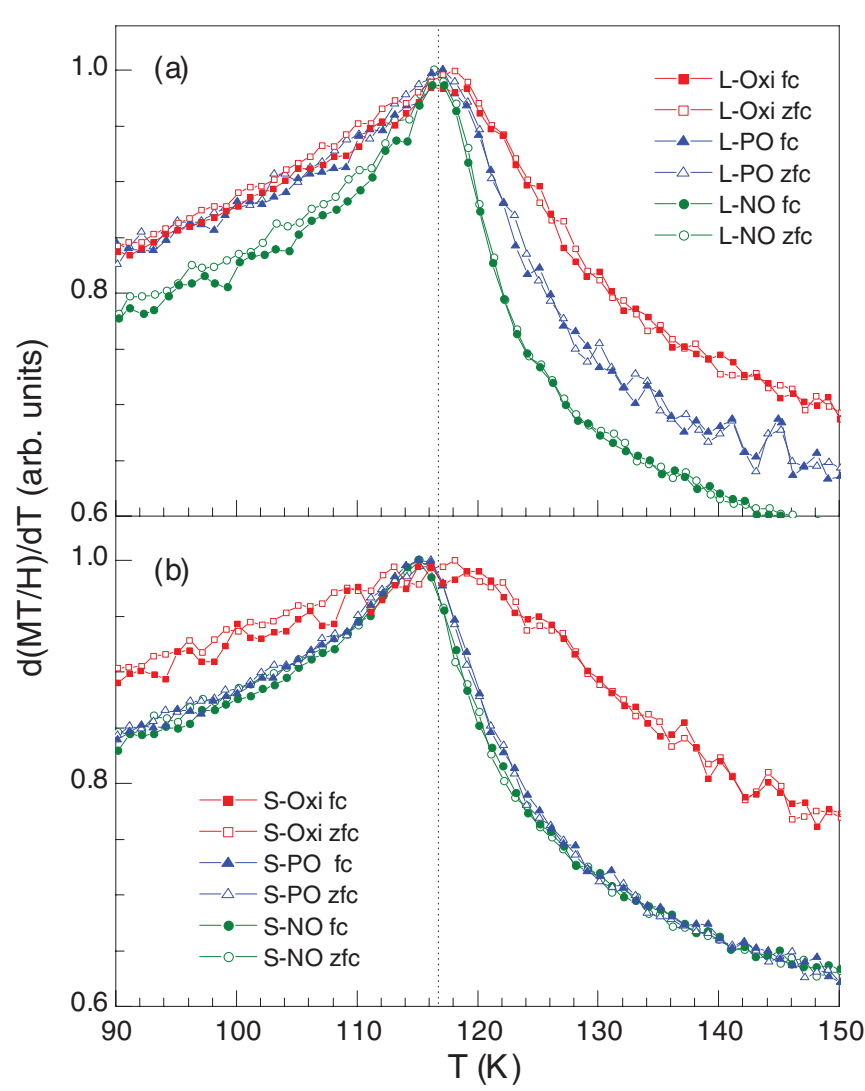

FIG. 5. (Color online) Temperature dependence of the normalized temperature derivative of the low-field susceptibility temperature product $d(M T / H) / d T$ of $\mathrm{L}$ samples [panel (a)] and S samples [panel (b)]. Vertical dotted line correspond to the maximum of $d(M T / H) / d T$ of sample L-NO.

the appearance of new degrees of freedom which interact with the AF order parameter, ${ }^{43}$ and (iii) size dependence of the lattice parameter. ${ }^{44}$ In the case of $\mathrm{MnO} / \mathrm{Mn}_{3} \mathrm{O}_{4}$ NPs in confining matrices, it was also suggested that the $T_{N}$ increase is a matrix effect, ${ }^{27,28}$ as a pressure effect induced by the matrix, or a geometry effect. ${ }^{28}$ However, not all authors report an increase of $T_{N}$. Ultrafine NPs $(3.7-5.4 \mathrm{~nm})$ showed a decrease of $T_{N}{ }^{45}$ while a recent report shows a slight increase of $T_{N}$ in $13 \mathrm{~nm} \mathrm{MnO} \mathrm{NPs}(120 \mathrm{~K})$ and a decrease of $T_{N}$ in $8 \mathrm{~nm}$ ones $(114 \mathrm{~K}) .{ }^{24}$ In this case, the difference was attributed to particle morphology, NPs/matrix interface, and to other magnetic species due to changes in the surface coordination number. According to the present results, the behavior of $T_{N}$ in $\mathrm{MnO} / \mathrm{Mn}_{3} \mathrm{O}_{4} \mathrm{NPs}$ is a combination of an increase imposed by oxidation and the decrease resulting from size decrease, the former being the dominant effect in larger NPs and the latter the dominant effect in ultrafine NPs.

A better insight on the structural changes induced by oxidation and on how oxidation leads to an increase of $T_{N}$ is obtained by the analysis of PND results. As expected, below $T_{N}$ the $\mathrm{MnO}$ nanoparticles have a magnetostructural transition, as shown by the split of nuclear peaks and the appearance of peaks associated with AF ordering (Fig. 6). The patterns of the nonoxidized samples can be refined to a cubic face centered cell (space group $F m \overline{3} m$ ) at high temperature, and to a rhombohedral nuclear cell (space group $R \overline{3} m$ ) and 


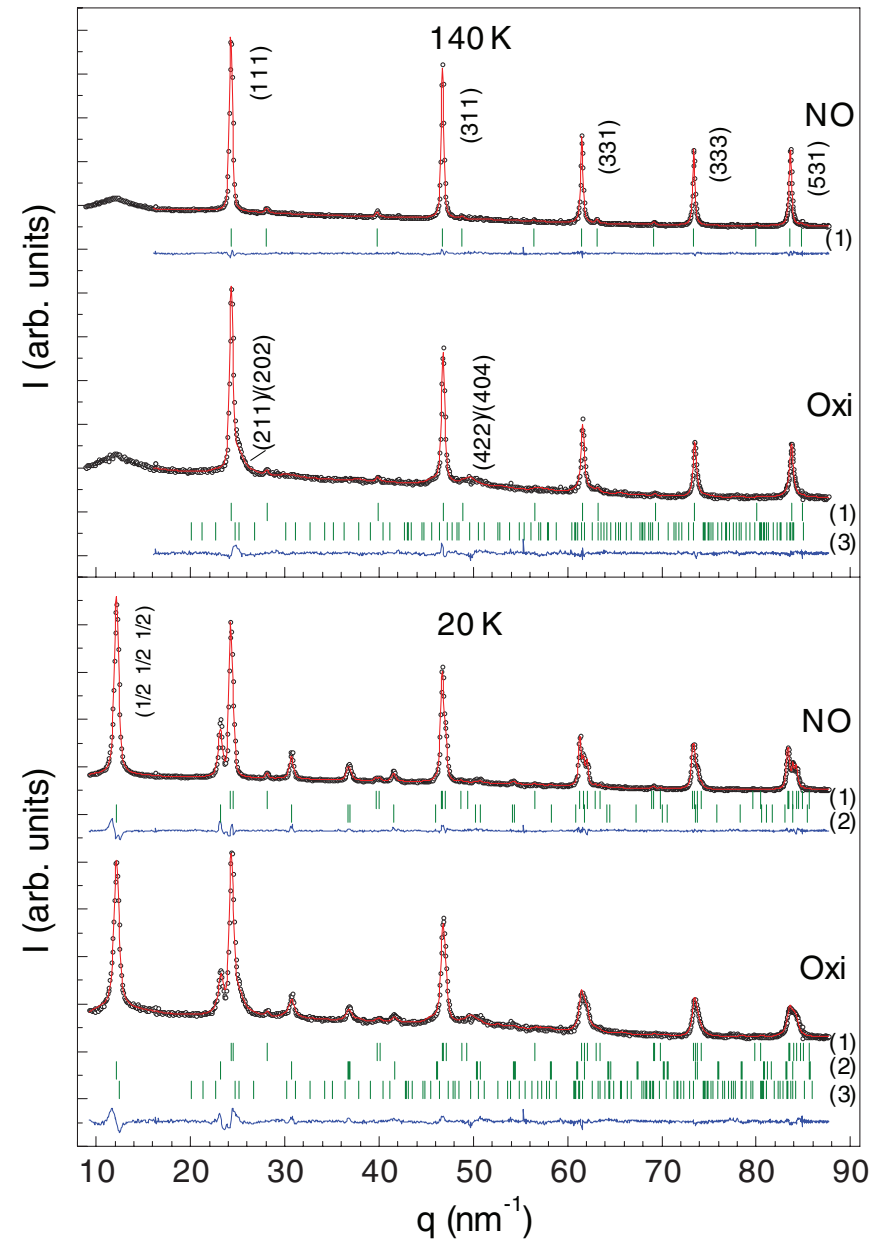

FIG. 6. (Color online) Powder neutron diffraction patterns of samples S-NO and S-Oxi at $140 \mathrm{~K}$ and at $20 \mathrm{~K}$, obtained at D20 powder diffractometer (ILL). Vertical (green) lines represent the position of the allowed Bragg peaks of nuclear (1) and magnetic (2) origin of $\mathrm{MnO}$ and nuclear origin of $\mathrm{Mn}_{3} \mathrm{O}_{4}$ (3). Continuous (red) lines correspond to Rietveld refinement and residues (blue, noise-like line).

by the propagation vector $\mathbf{k}=[0,0,3 / 2]$ referred to the rhombohedral cell (which corresponds to the $[1 / 2,1 / 2,1 / 2]$ propagation vector referred to a distorted cubic cell) at low temperature. The rhombohedral structure can be described in terms of a pseudocubic cell with a cell edge $a_{p c}$ and an angle between edges of $90+\Delta$ (degrees) ${ }^{46,47}$ In the oxidized samples, broad and small shoulders appear at $q \simeq 25 \mathrm{~nm}^{-1}$ and $q \simeq 50 \mathrm{~nm}^{-1}$, corresponding to the most important features of $\mathrm{Mn}_{3} \mathrm{O}_{4}[(211) /(202)$ and (422)/(404) planes, respectively, space group $\left.I 4_{1} / a m d\right] .^{48}$ In the studied temperature range, the magnetic peak at $\sim 12 \mathrm{~nm}^{-1}$ has two components, a narrower and more intense one decreasing with temperature up to $\sim 120 \mathrm{~K}$ and a broader and less intense scattering nearly constant in the studied temperature range, in accordance with early neutron diffraction results. ${ }^{46}$ Recently, polarized neutron scattering data showed that the latter component is an incoherent scattering associated with the presence of disordered spins. ${ }^{24}$

It is clear from Fig. 7(a) that oxidation reduces the cell parameter $a_{p c}$ this effect being more dramatic in the $\mathrm{S}$ samples.

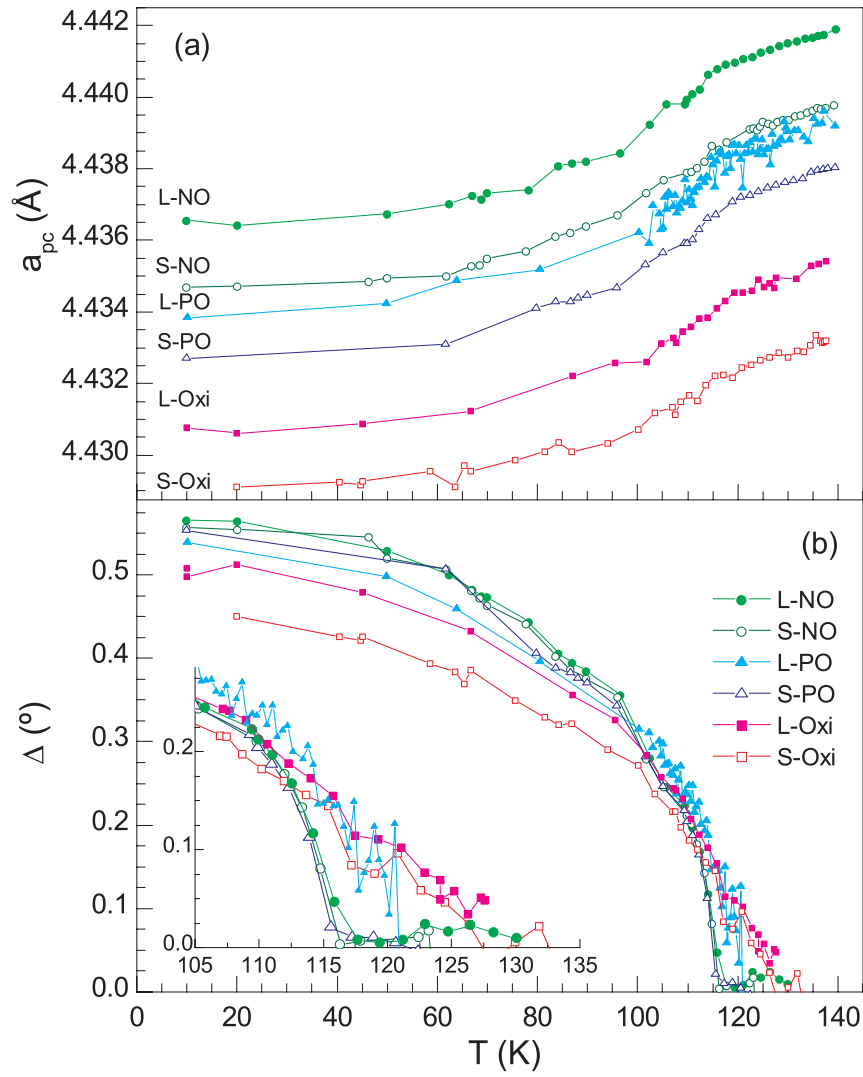

FIG. 7. (Color online) Temperature dependence of the structural parameters of the (pseudo) cubic cell $a_{p c}$ [panel (a)] and $\Delta$ [panel (b)]. Inset in panel (b) shows zoom over high-temperature region.

This can be understood considering the fact that $\mathrm{Mn}_{3} \mathrm{O}_{4}$ grows epitaxially on $\mathrm{MnO}^{22}$ and that the $\mathrm{MnO} / \mathrm{Mn}_{3} \mathrm{O}_{4}$ lattice mismatch is such that the $\mathrm{MnO}$ lattice is larger than the $\mathrm{Mn}_{3} \mathrm{O}_{4}$ one. As shown in Ref. 22, growth can occur on the (111) $\mathrm{MnO}$ plane, which by $\mathrm{Mn}^{2+}$ vacancies adopts an atomic coordination similar to that of the (202) plane (space group I4 1 /amd) of $\mathrm{Mn}_{3} \mathrm{O}_{4}$ and with higher interatomic distances in $\mathrm{MnO}(3.143 \AA$ versus 3.121 and $2.882 \AA$ in $\mathrm{Mn}_{3} \mathrm{O}_{4}$ ). At low temperature, the distortion angle $\Delta$ decreases with the increase of oxidation [Fig. 7(b)]. At the same time, for nonoxidized samples $\Delta$ shows a relatively sharp transition near $117 \mathrm{~K}$ (in agreement with the magnetometry results), while for oxidized samples $\Delta$ approaches zero more smoothly and at higher temperatures. The behavior of $\Delta(T)$ is also consistent with a small decrease of the transition temperature with size when comparing the nonoxidized samples.

The average apparent size of the $\mathrm{MnO}$ nuclear ordering of $\mathrm{L}$ samples is higher than that of $\mathrm{S}$ samples and constant with temperature being of the order of those obtained from TEM. The magnetic ordering average apparent size is about half of the nuclear one, suggesting that in average MnO NPs are magnetic bi-domains. With oxidation, the nuclear average apparent size of the $\mathrm{L}$ samples shows a decrease of the order of the $\mathrm{Mn}_{3} \mathrm{O}_{4}$ shell size, while in $\mathrm{S}$ samples no decrease is observed [Fig. 8(a)]. On the other hand, the effect of oxidation is quite pronounced in strain, which increases about 2 and 3 times [Fig. 8(b)] being more intense in the smaller sample. The increase of strain is consistent with the observed decrease of 


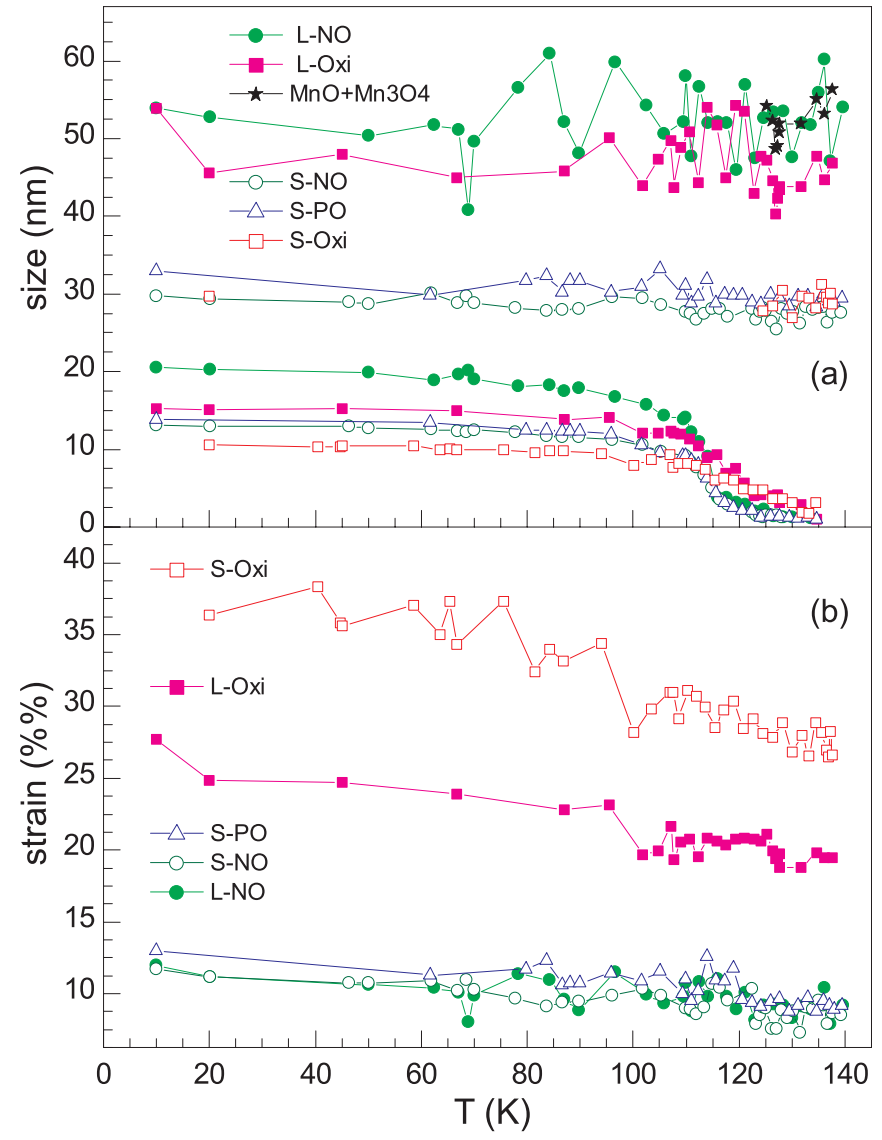

FIG. 8. (Color online) Panel (a): Temperature dependence of the average apparent size of the $\mathrm{MnO}$ nuclear ordering (curves constant with temperature) and of the average apparent size of the $\mathrm{MnO}$ magnetic ordering (curves decreasing to zero at high temperature). The sum of the $\mathrm{MnO}$ and $\mathrm{Mn}_{3} \mathrm{O}_{4}$ average apparent sizes of sample L-Oxi at high temperature is also shown. Panel (b): Temperature dependence of the average maximum strain.

$a_{p c}$ with oxidation induced by the epitaxial growth of $\mathrm{Mn}_{3} \mathrm{O}_{4}$ on the MnO surface. All samples show a decrease of strain with the increase of temperature, probably associated with the magnetic-induced structural distortion that decreases with increasing temperature due to the decrease of the AF sublattice magnetic moment $\mu .{ }^{49}$

The low temperature $\mu$ of NO samples is close to that reported for bulk (bulk values oscillate from $\mu=4.58(6) \mu_{B}{ }^{38}$ to $\mu=4.8 \pm 0.3 \mu_{B}{ }^{24}$ ), decreasing in PO and Oxi samples, due to disorder induced by oxidation (Fig. 9). Again, the decrease of the low-temperature $\mu$ in $\mathrm{MnO} / \mathrm{Mn}_{3} \mathrm{O}_{4}$ NPs is not a pure finite-size or a pure surface effect but rather a shell-induced effect. This effect can also be observed in the decrease of the low-temperature $\Delta$ with oxidation, since $\mu$ is proportional to $\sqrt{\Delta} \cdot{ }^{47,49}$ As observed in the case of $\Delta$, the $\mu$ approach to zero is smoother in oxidized samples and their $T_{N}$ is higher. Between 120 and $140 \mathrm{~K}$ the intensity of the magnetic peak is of the order of noise hindering the determination of $\mu$ and in particular the determination of the temperature at which $\mu$ is zero. The decrease of $\mu$ with temperature is followed by the decrease of the average apparent size of the magnetic domains [Fig. 8(a)]. Again, the decrease of this size in the

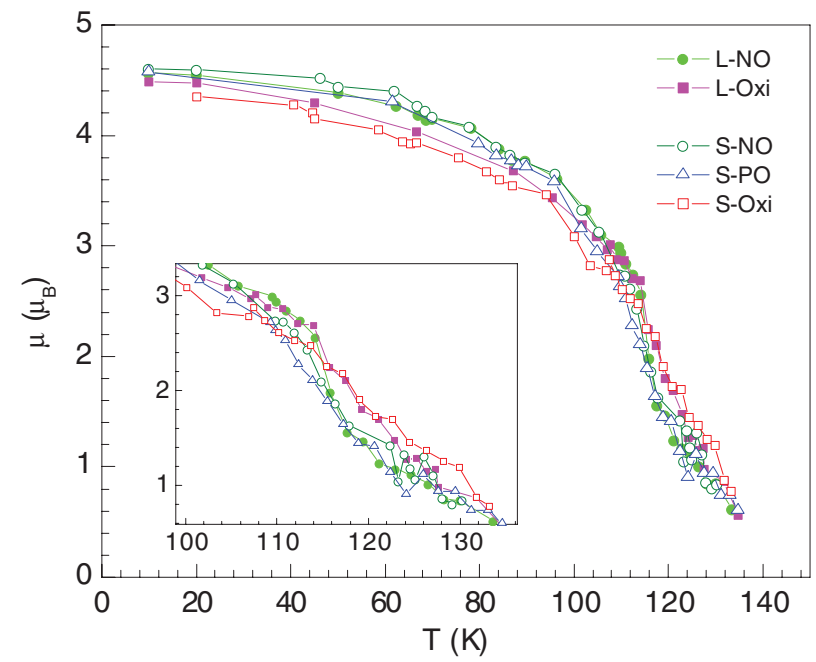

FIG. 9. (Color online) Temperature dependence of the sublattice magnetic moment $\mu$. Inset shows a zoom over the high-temperature region.

oxidized samples is smoother and small magnetically ordered domains survive up to higher temperature.

At this point, it is clear that oxidation has an influence on both structural and magnetic properties of MnO NPs, inducing an increase of $T_{N}$ and a reduction in the lattice size with a concomitant increase of strain. The relation between structural and magnetic changes can be observed in Fig. 10, where $T_{N}$ determined by $\Delta(T) \simeq 0$ is plotted as a function of $a_{p c}$ at $140 \mathrm{~K}$. Due to the broadening of the transition induced by oxidation, $T_{N}$ values thus determined are larger than those determined from the temperature at which $d(M T / H) / d T$

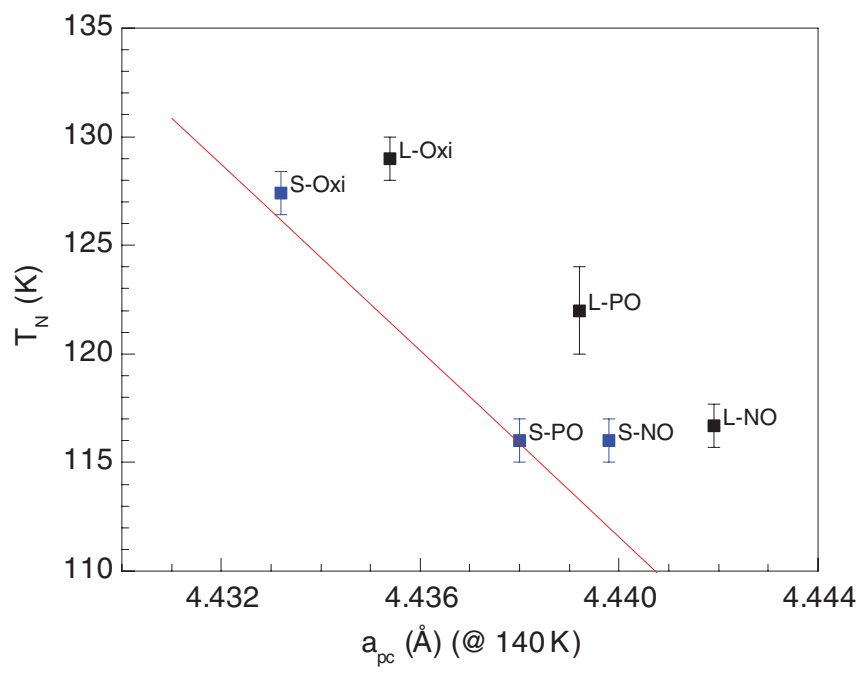

FIG. 10. (Color online) $T_{N}$ as a function of the cell parameter $a_{p c}$ determined at $140 \mathrm{~K}$ for the studied samples. Line represent the expected dependence of $T_{N}$ with $a_{p c}$ in bulk $\mathrm{MnO}$ where $a_{p c}$ is changed by an external pressure $p$. This dependence was obtained by comparing the $T_{N}(p)$ dependence of Ref. 44 with the normalized $a_{p c}(p) / a_{p c}(0)$ dependence obtained from the $V(p)$ dependence of Refs. 50,51, considering $a_{p c} \propto V^{1 / 3}$ and eliminating the pressure parameter to obtain $T_{N}\left(a_{p c}\right)$ at room temperature. Data was then scaled to the bulk $a_{p c}$ value at $140 \mathrm{~K}$ (Ref. 47). 
is maximum. In both $\mathrm{L}$ and $\mathrm{S}$ series, oxidation leads to a decrease of $a_{p c}$ resulting in an increase of $T_{N}$, while the $T_{N}$ of $S$ samples are shifted to lower values; i.e., for a given $a_{p c}$, the corresponding $T_{N}$ is lower in $S$ samples, probably because of size effects.

Moreover, the trend is similar to the effect of an external pressure on bulk $\mathrm{MnO}$ experimentally observed for pressures below $\sim 1 \mathrm{GPa} .{ }^{50,51}$ The $a_{p c}$ for bulk at room pressure and $140 \mathrm{~K}$ is lower than that of the present samples, which may reflect a small systematic difference in our determination of $a_{p c}$ compared to that of Ref. 47. The effects of an external pressure in the structural and magnetic properties of bulk $\mathrm{MnO}$ are quite complex, involving distinct order parameters and their coupling with strains, ${ }^{52}$ and thus shell-induced pressure effects on MnO NPs are also expected to be complex. However, the changes in $T_{N}$ are well understood, in first approach, in terms of the magnetic Gruneisen parameter $\gamma_{m} \equiv-\partial \ln T_{N} / \partial \ln a_{p c}$ with $\gamma_{m}=68$ in L and S series compared to $\gamma_{m}=65$ found in bulk-pressure dependence results, being higher than that found in $\mathrm{CuO}\left(\gamma_{m}=30\right) .{ }^{53}$ The increase of $T_{N}$ with the decrease of $a_{p c}$ is also in accordance with theoretical calculations that show the increase of the modulus of the exchange constants with the decrease of $a_{p c} .{ }^{54}$

\section{CONCLUSIONS}

In summary, the effects of size and oxidation on the properties of $\mathrm{MnO} / \mathrm{Mn}_{3} \mathrm{O}_{4} \mathrm{NPs}$ were independently addressed. It was shown that surface oxidation is responsible for the often observed increase of $T_{N}$ in MnO NPs, due to an effect of shell strain, resulting in pressure effect on the $\mathrm{MnO}$ core. This effect is balanced by the finite-size effect that tends to decrease $T_{N}$ with the decrease of size. The final $T_{N}$ value results from the balance between oxidation and size effects, this being the most probable origin of the dispersion of values found in literature. This highlights the possible control of the magnetic properties of $\mathrm{MnO}$ by the control of size and oxidation.

\section{ACKNOWLEDGMENT}

The authors thank Thomas Hansen and Susana Braga for their collaboration in the experiments at D20, Noemi Carmona for supplying the $\mathrm{Mn}_{3} \mathrm{O}_{4}$ XANES spectra, and Juan Rodríguez-Carvajal for enlightening discussions. The authors thank ILL, SpINS, and SpLine for beamtime allocation and IFIMUP-IN (Universidade do Porto) for access to the magnetometer laboratory. The Aveiro-Zaragoza collaboration has been supported by the Integrated SpanishPortuguese Action PT2009-0131. The work in Zaragoza has been supported by Research Grants No. MAT201127233-C02-02, No. MAT2011-25991, and CONSOLIDER CSD2007-00010 from the Ministry of Education. The financial support of the CSIC and Spanish Ministerio de Ciencia e Innovación (PI201060E013) is also acknowledged. The work in Aveiro has been supported by Fundação para a Ciência e Tecnologia, COMPETE, and FEDER programs (Pest-C/CTM/LA0011/2011). N.J.O.S. acknowledges FCT for the Ciência 2008 program. *nunojoao@ua.pt

${ }^{1}$ R. Ghosh Chaudhuri and S. Paria, Chem. Rev. 112, 2373 (2012).

${ }^{2}$ A. Tarun, N. Hayazawa, H. Ishitobi, S. Kawata, M. Reiche, and O. Moutanabbir, Nano Lett. 11, 4780 (2011).

${ }^{3}$ M. Ieong, B. Doris, J. Kedzierski, K. Rim, and M. Yang, Science 306, 2057 (2004).

${ }^{4}$ R. D. Robinson, B. Sadtler, D. O. Demchenko, C. K. Erdonmez, L.-W. Wang, and A. P. Alivisatos, Science 317, 355 (2007).

${ }^{5}$ F. Banhart, E. Hernández, and M. Terrones, Phys. Rev. Lett. 90, 185502 (2003).

${ }^{6}$ V. C. Holmberg, M. G. Panthani, and B. A. Korgel, Science 326, 405 (2009).

${ }^{7}$ I. L. Liu, B. C. Lin, S. Y. Chen, and P. Shen, J. Phys. Chem. C 115, 4994 (2011).

${ }^{8}$ X. Chen, Y. Lou, A. C. Samia, and C. Burda, Nano Lett. 3, 799 (2003).

${ }^{9}$ A. M. Smith, A. M. Mohs, and S. Nie, Nat. Nanotechnol. 4, 56 (2009).

${ }^{10}$ Z. Gu, L. Zou, Z. Fang, W. Zhu, and X. Zhong, Nanotechnology 19, 135604 (2008).

${ }^{11}$ M. Montazeri, M. Fickenscher, L. M. Smith, H. E. Jackson, J. Yarrison-Rice, J. H. Kang, Q. Gao, H. H. Tan, C. Jagadish, Y. Guo et al., Nano Lett. 10, 880 (2010).

${ }^{12}$ P. Ghosh, J. Oliva, E. D. 1. Rosa, K. K. Haldar, D. Solis, and A. Patra, J. Phys. Chem. C 112, 9650 (2008).

${ }^{13}$ V. Lopez-Richard, J. C. González, F. M. Matinaga, C. TralleroGiner, E. Ribeiro, M. R. Sousa Dias, L. Villegas-Lelovsky, and G. E. Marques, Nano Lett. 9, 3129 (2009).
${ }^{14}$ J. Lee, H. Kim, S. Kahng, G. Kim, Y. Son, J. Ihm, H. Kato, Z. Wang, T. Okazaki, H. Shinohara et al., Nature (London) 415, 1005 (2002).

${ }^{15}$ R. Jacobsen, K. Andersen, P. Borel, J. Fage-Pedersen, L. Frandsen, O. Hansen, M. Kristensen, A. Lavrinenko, G. Moulin, H. Ou et al., Nature (London) 441, 199 (2006).

${ }^{16}$ Y. S. Koo, K. M. Song, N. Hur, J. H. Jung, T. H. Jang, H. J. Lee, T. Y. Koo, Y. H. Jeong, J. H. Cho, and Y. H. Jo, Appl. Phys. Lett. 94, 032903 (2009).

${ }^{17}$ H. Zheng, J. Kreisel, Y.-H. Chu, R. Ramesh, and L. SalamancaRiba, Appl. Phys. Lett. 90, 113113 (2007).

${ }^{18}$ M. Feygenson, Y. Yiu, A. Kou, K.-S. Kim, and M. C. Aronson, Phys. Rev. B 81, 195445 (2010).

${ }^{19}$ Y. Nahas, V. Repain, C. Chacon, Y. Girard, J. Lagoute, G. Rodary, J. Klein, S. Rousset, H. Bulou, and C. Goyhenex, Phys. Rev. Lett. 103, 067202 (2009).

${ }^{20}$ S. Zhou, K. Potzger, J. von Borany, R. Grötzschel, W. Skorupa, M. Helm, and J. Fassbender, Phys. Rev. B 77, 035209 (2008).

${ }^{21}$ J. Park, E. Kang, C. J. Bae, J.-G. Park, H.-J. Noh, J.-Y. Kim, J.-H. Park, H. M. Park, and T. Hyeon, J. Phys. Chem. B 108, 13594 (2004).

${ }^{22}$ A. E. Berkowitz, G. F. Rodriguez, J. I. Hong, K. An, T. Hyeon, N. Agarwal, D. J. Smith, and E. E. Fullerton, Phys. Rev. B 77, 024403 (2008)

${ }^{23}$ I. V. Golosovsky, G. Salazar-Alvarez, A. López-Ortega, M. A. González, J. Sort, M. Estrader, S. Suriñach, M. D. Baró, and J. Nogués, Phys. Rev. Lett. 102, 247201 (2009). 
${ }^{24}$ C. H. Wang, S. N. Baker, M. D. Lumsden, S. E. Nagler, W. T. Heller, G. A. Baker, P. D. Deen, L. M. D. Cranswick, Y. Su, and A. D. Christianson, Phys. Rev. B 83, 214418 (2011).

${ }^{25}$ G. Salazar-Alvarez, J. Sort, S. Suriñach, M. D. Baró, and J. Nogués, J. Am. Chem. Soc. 129, 9102 (2007).

${ }^{26}$ A. López-Ortega, D. Tobia, E. Winkler, I. V. Golosovsk, G. Salazar-Alvarez, S. Estradé, M. Estrader, J. Sort, M. A. González, S. Suriñach et al., J. Am. Chem. Soc. 132, 9398 (2010).

${ }^{27}$ M. Feygenson, W. Schweika, A. Ioffe, S. B. Vakhrushev, and T. Brückel, Phys. Rev. B 81, 064423 (2010).

${ }^{28}$ I. V. Golosovsky, I. Mirebeau, G. André, D. A. Kurdyukov, Y. A. Kumzerov, and S. B. Vakhrushev, Phys. Rev. Lett. 86, 5783 (2005).

${ }^{29}$ I. V. Golosovsky, I. Mirebeau, V. P. Sakhnenko, D. A. Kurdyukov, and Y. A. Kumzerov, Phys. Rev. B 72, 144409 (2005).

${ }^{30}$ I. V. Golosovsky, I. Mirebeau, F. Fauth, M. Mazaj, D. A. Kurdyukov, and Y. A. Kumzerov, Phys. Rev. B 74, 155440 (2006).

${ }^{31}$ D. Zitoun, N. Pinna, N. Frolet, and C. Belin, J. Am. Chem. Soc. 127, 15034 (2005).

${ }^{32}$ T. Ould-Ely, D. Prieto-Centurion, A. Kumar, W. Guo, W. V. Knowles, S. Asokan, M. S. Wong, I. Rusakova, A. Lüttge, and K. H. Whitmire, Chem. Mater. 18, 1821 (2006).

${ }^{33}$ M. Yin and S. O’Brien, J. Am. Chem. Soc. 125, 10180 (2003).

${ }^{34}$ N. R. Jana, Y. Chen, and X. Peng, Chem. Mater. 16, 3931 (2004).

${ }^{35}$ Q.-C. Sun, X. Xu, S. N. Baker, A. D. Christianson, and J. L. Musfeldt, Chem. Mater. 23, 2956 (2011).

${ }^{36}$ B. Ravel and M. Newville, J. Synchrotron Radiat. 12, 537 (2005).

${ }^{37}$ J. Rodríguez-Carvajal, Physica B 192, 55 (1993).
${ }^{38}$ A. K. Cheetham and D. A. O. Hope, Phys. Rev. B 27, 6964 (1983).

${ }^{39}$ Y.-F. Han, K. Ramesh, L. W. Chen, F. X. Chen, and A. Borgna, Adv. Syn. Rad. 1, 6778 (2008).

${ }^{40}$ L. J. De Jongh and A. R. Miedema, Adv. Phys. 23, 1 (1974).

${ }^{41}$ S. Todd and K. R. Bonnickson, J. Am. Chem. Soc. 73, 3894 (1951).

${ }^{42}$ J. Wesselinowa, J. Magn. Magn. Mater. 322, 234 (2010).

${ }^{43}$ M. A. Morales, R. Skomski, S. Fritz, G. Shelburne, J. E. Shield, M. Yin, S. O’Brien, and D. L. Leslie-Pelecky, Phys. Rev. B 75, 134423 (2007).

${ }^{44}$ I. Kantor, L. Dubrovinsky, C. Mccammon, N. Dubrovinskaia, I. Goncharenko, A. Kantor, A. Kuznetsov, and W. Crichton, Phase Transitions 80, 1151 (2007).

${ }^{45}$ S. Sako, Y. Umemura, K. Ohshima, M. Sakai, and S. Bandow, J. Phys. Soc. Jpn. 65, 280 (1996).

${ }^{46}$ W. L. Roth, Phys. Rev. 110, 1333 (1958).

${ }^{47}$ B. Morosin, Phys. Rev. B 1, 236 (1970).

${ }^{48}$ D. Jarosch, Min. Petr. 37, 15 (2005).

${ }^{49}$ D. S. Rodbell and J. Owen, J. Appl. Phys. 35, 1002 (1964).

${ }^{50}$ T. Kondo, T. Yagi, Y. Syono, Y. Noguchi, T. Atou, T. Kikegawa, and O. Shimomura, J. Appl. Phys. 87, 4153 (2000).

${ }^{51}$ R. Jeanloz and A. Rudy, J. Geophys. Res. 92, 11433 (1987).

${ }^{52}$ M. A. Carpenter, Z. Zhang, and C. J. Howard, J. Phys.: Condens. Matter 24, 156002 (2012).

${ }^{53}$ A. Punnoose, H. Magnone, M. S. Seehra, and J. Bonevich, Phys. Rev. B 64, 174420 (2001).

${ }^{54}$ G. Fischer, M. Däne, A. Ernst, P. Bruno, M. Lüders, Z. Szotek, W. Temmerman, and W. Hergert, Phys. Rev. B 80, 014408 (2009). 